\title{
Understanding the Association Pattern of Body Mass Index with Risk of Mortality among Participants with Diabetes
}

\author{
Kayoung Lee* \\ Department of Family Medicine, Inje University Busan Paik Hospital, Inje University College of Medicine \\ Busan, Korea
}

\author{
Received October 5, 2018 \\ Reviewed November 2, 2018 \\ Accepted November 9, 2018 \\ ${ }^{*}$ Corresponding author \\ Kayoung Lee \\ https://orcid.org/0000-0002-2816-554X \\ Department of Family Medicine, Inje \\ University Busan Paik Hospital, Inje \\ University College of Medicine, \\ 75 Bokji-ro, Busanjin-gu, Busan 47392, \\ Korea \\ Tel: $+82-51-890-6229$ \\ Fax: +82-51-894-7554 \\ E-mail: kayoung.fmlky@gmail.com
}

Weight loss is recommended for overweight or obese adults with type 2 diabetes mellitus (T2DM) with "a evidence." ${ }^{1}$ However, evidence on the association between obesity and mortality in patients with diabetes remains controversial. Previous studies of body mass index (BMI) and mortality among individuals with diabetes suggested inverse ${ }^{2}$, J- or U-shaped ${ }^{3}$, or linear associations. ${ }^{4}$ Recently, two systemic review and meta-analysis studies investigating the relationship between BMI and all-cause mortality in patients with T2DM found a nonlinear association. ${ }^{5,6}$ Kwon et al..$^{5}$ demonstrated U-shaped relationship with a BMI nadir of $28-30 \mathrm{~kg} / \mathrm{m}^{2}$ in the meta-analysis including 16 cohort studies. Likewise, Zaccardi et al. ${ }^{6}$ reported a nonlinear relationship between BMI and all-cause mortality in both men and women, with a BMI nadir of $31-35 \mathrm{~kg} / \mathrm{m}^{2}$ and $28-31 \mathrm{~kg} / \mathrm{m}^{2}$, respectively in a systemic review including 21 cohort studies. Based on these meta-analysis findings, obese individuals with T2DM may have better outcomes than their counterparts with normal weight. Thus, "obesity paradox" may exist in this association.

Lee et al. ${ }^{7}$ examined the association of BMI with the risk of major adverse cardiovascular events (MACE) and all-cause mortality in 48,438 participants (male, $58.1 \%$; median age, 56.1 years) with diabetes from the Korean National Health Insurance Service-National Health Screening Cohort from 2002 to 2003. Median followup was 10.7 years, during which 7,360 MACE and 5,766 deaths occurred. Compared to those with normal weight (BMI, 18.5$22.9 \mathrm{~kg} / \mathrm{m}^{2}$ ), those with overweight (BMI, $23.0-24.9 \mathrm{~kg} / \mathrm{m}^{2}$ ) and obesity class I (BMI, 25.0-29.9 kg/m²) had respectively $9 \%$ and $7 \%$ lower risk for MACE and 26\% and 33\% lower risk for all-cause mortality. Even when deaths occurring less than 3 years after the index date were excluded, individuals with underweight (BMI $<18.5 \mathrm{~kg} / \mathrm{m}^{2}$ ) had $65 \%$ higher risk for all-cause mortality, while those with overweight, obesity class I, or obesity class II (BMI $\geq 30$ $\mathrm{kg} / \mathrm{m}^{2}$ ) had respectively $25 \%, 31 \%$, and $23 \%$ lower risk for all-cause mortality compared to those with normal weight. Risk of cardiovascular death was 1.5 times higher in individuals with underweight, while $20 \%$ and $23 \%$ lower in those with overweight and obesity class I, respectively. In the subgroup analyses, this association pattern of BMI categories with all-cause mortality was consistent regardless of age groups, smoking status, concomitant hypertension, or use of hypoglycemic medication.

This pattern of all-cause mortality with respect to BMI categories in Korean patients with diabetes suggests that overweight or obesi- 
ty in patients with diabetes may be beneficial regarding risk of allcause mortality compared to lean or normal weight patients. Therefore, the findings of Lee et al's study ${ }^{7}$ may also reflect "obesity paradox." However, these findings should be cautiously interpreted. Authors addressed several limitations such as potential of inaccurate diagnoses, non-differentiation of diabetes types, and incomplete control of confounding factors including weight change, central obesity, duration of diabetes, and severity of diabetes. Not only these limitations, other factors influencing on reverse-causation such as undiagnosed chronic diseases and the order of BMI measurement and diabetes diagnosis ${ }^{4}$ were not taken into account. The obesity paradox may be explained by several possibilities. Patients who are underweight or with normal weight may have long-standing diabetes compared to their overweight or obese counterparts and then, be at higher risk for poorer prognosis. ${ }^{5}$ Individuals with higher genetic predisposition to diabetes may be more prone to developing diabetes and diabetes complications at lower BMI categories. ${ }^{2}$ Different adiposity types may play a role in these mechanisms. Individuals with a higher BMI with lower mortality may be physically fit with highly functional adiposity types. ${ }^{8}$ Given evidence for $\mathrm{BMI}$ ranges for lowest mortality in patients with diabetes, management strategies for normal weight and underweight patients with diabetes need to focus on improving cardiorespiratory fitness and maintaining their weight status.

\section{CONFLICTS OF INTEREST}

The author declares no conflict of interest.

\section{REFERENCES}

1. American Diabetes Association. 4. Lifestyle management: standards of medical care in diabetes-2018. Diabetes Care 2018;41 (Suppl 1):S38-50.

2. Carnethon MR, De Chavez PJ, Biggs ML, Lewis CE, Pankow JS, Bertoni AG, et al. Association of weight status with mortality in adults with incident diabetes. JAMA 2012;308:581-90.

3. Logue J, Walker JJ, Leese G, Lindsay R, McKnight J, Morris A, et al. Association between BMI measured within a year after diagnosis of type 2 diabetes and mortality. Diabetes Care 2013; 36:887-93.

4. Tobias DK, Pan A, Jackson CL, O’Reilly EJ, Ding EL, Willett WC, et al. Body-mass index and mortality among adults with incident type 2 diabetes. N Engl J Med 2014;370:233-44.

5. Kwon Y, Kim HJ, Park S, Park YG, Cho KH. Body mass index-related mortality in patients with type 2 diabetes and heterogeneity in obesity paradox studies: a dose-response metaanalysis. PLoS One 2017;12:e0168247.

6. Zaccardi F, Dhalwani NN, Papamargaritis D, Webb DR, Murphy GJ, Davies MJ, et al. Nonlinear association of BMI with all-cause and cardiovascular mortality in type 2 diabetes mellitus: a systematic review and meta-analysis of 414,587 participants in prospective studies. Diabetologia 2017;60:240-8.

7. Lee DH, Ha KH, Kim HC, Kim DJ. Association of body mass index with risk of major adverse cardiovascular events and mortality in people with diabetes. J Obes Metab Syndr 2018; 27:61-70.

8. Goyal A, Nimmakayala KR, Zonszein J. Is there a paradox in obesity? Cardiol Rev 2014;22:163-70. 